\title{
$\varepsilon$-Nash equilibrium in stochastic differential games with mean-field interaction and controlled jumps
}

\author{
Chiara Benazzoli ${ }^{\mathrm{a}}$, Luciano Campi $^{\mathrm{b}}$, Luca Di Persio ${ }^{\mathrm{c}}$ \\ ${ }^{a}$ Department of Mathematics, University of Trento, Italy \\ ${ }^{b}$ Department of Statistics, London School of Economics, UK \\ ${ }^{c}$ Department of Computer Science, University of Verona, Italy
}

\begin{abstract}
We consider a symmetric $n$-player nonzero-sum stochastic differential game with jump-diffusion dynamics and mean-field type interaction among the players. Under the assumption of existence of a regular Markovian solution for the corresponding limiting mean-field game, we construct an approximate Nash equilibrium for the $n$-player game for $n$ large enough, and provide the rate of convergence. This extends to a class of games with jumps classical results in mean-field game literature. This paper complements our previous work [2] on the existence of solutions of mean-field games for jump-diffusions.
\end{abstract}

Keywords: stochastic differential games, Nash equilibrium, mean-field games, marked point processes, jump measures.

\section{Introduction}

In this paper we consider a symmetric nonzero-sum stochastic differential game with jump-diffusion dynamics, where the interaction among the players is of mean-field type. Mean-field games (MFGs, henceforth) are optimization problems that were simultaneously introduced by Lasry and Lions in $[16,17,18]$ and by Huang and co-authors in [13]. They can be seen as an approximation of large population symmetric stochastic differential games, whose players interact via the empirical law of their private states. When the number $n$ of players is large enough, a solution of the limit MFG can be used to provide nearly Nash equilibria for the corresponding $n$-player games, see, e.g., $[3,4,5,6,13,15]$. The importance of MFG lies also in the fact that computing Nash equilibria in $n$-player games, when $n$ is large, is usually not feasible, even numerically, because of the curse of dimensionality. Moreover, MFGs represent a very flexible framework for applications spanning from finance to economics and crowd dynamics, see, e.g., $[5,11]$. In the 
symmetric stochastic differential game we consider in this paper, the agents interact through the empirical distribution of their private states, affecting all coefficients in the jump-diffusion dynamics. According to MFG theory, we expect that as the number of players gets very large, the $n$-player games tend to the MFG with jumps studied in [2].

Our main contribution is that any solution to the limiting MFG, provided it is Markovian and Lipschitz continuous in the state variable, gives a good approximation of Nash equilibria in the $n$-player game. This result extends to a jump-diffusion setting classical results proved for continuous paths state variables as in, e.g., [5, Vol. II] and references therein. From the application viewpoint, the presence of jumps allows to model unpredictable exogenous shocks affecting the state variable. This additional feature can be important especially for applications in economics and finance (see, e.g., [2]). Indeed, jumps are very natural tools to model exogenous unexpected shocks. Regarding the literature on the topic, while the uncontrolled counter-part of MFG, namely particle systems and propagation of chaos for jump processes, has been thoroughly studied in the probabilistic literature, see, e.g., $[1,10,14]$, MFGs with jumps have attracted less attention, with the exception of few papers as [7, 9, 12, 15], among which only [12] deals with jump-diffusions state variables.

The paper is organized as follows: in Section 2 we describe the $n$-player game and the corresponding MFG. Section 3 contains the main result of this paper, establishing how under suitable conditions a Markovian MFG solution yields a nearly Nash equilibrium for the $n$-player game with $n$ sufficiently large.

Finally, notice that our setting is one-dimensional only for the sake of simplicity, extending our results to a multi-dimensional state space is straightforward.

\section{A symmetric $n$-player game with interaction of mean-field type}

In this section we describe the $n$-player game we are interested in, together with the corresponding MFG, we set the main assumptions and we provide some a-priori estimates on the state variables. In the sequel, $\mathcal{L}(X)$ denotes the law of some random variable $X$, while $\mathcal{P}(\mathbb{R})\left(\operatorname{resp} . \mathcal{P}^{q}(\mathbb{R}), q \geq 1\right)$ will denote the set of all probability measures on the real line equipped with the Borel $\sigma$-field (resp. with finite moment of order $q$ ). Moreover, the notation $\|\cdot\|_{L^{q}}$ for the $L^{q}(P)$-norm will be used to shorten the formulae when necessary. 


\subsection{The n-player game $G_{n}$ with mean-field interaction.}

Let $\left(\Omega, \mathcal{F},\left(\mathcal{F}_{t}\right)_{t \in[0, T]}, \mathbb{P}\right)$ be a filtered probability space satisfying the usual conditions, supporting $n$ independent Brownian motions $W^{i}$ and $n$ independent marked point processes $\mu^{i}$, all with predictable intensity kernel $\nu$ of the form $\nu_{t}(d z)=\lambda_{t} \Phi(d z)$, where $\Phi$ is a probability measure on $\mathbb{R}$. We note $\widetilde{\mu}^{i}(d z, d t)=\mu^{i}(d z, d t)-\nu_{t}(d z) d t$ the compensated jump measure. Let $X^{i, n}=X^{i, n}(\gamma)$ be the unique strong solutions to the following SDEs (conditions ensuring this will be given below)

$$
\begin{aligned}
d X_{t}^{i, n}= & b\left(t, X_{t}^{i, n}, \mu_{t}^{n}, \gamma_{t}^{i}\right) d t+\sigma\left(t, X_{t}^{i, n}, \mu_{t}^{n}, \gamma_{t}^{i}\right) d W_{t}^{i} \\
& +\int_{\mathbb{R}} \beta\left(t, X_{t-}^{n, i}, \mu_{t-}^{n}, \gamma_{t}^{i}, z\right) \widetilde{\mu}^{i}(d z, d t),
\end{aligned}
$$

with initial condition $X_{0}^{i, n}=\xi^{i}$, where $\left(\xi^{i}\right)_{i=1}^{n}$ are i.i.d. random variables with distribution $\chi$. Moreover, $\mu_{t}^{n}$ denotes the empirical distribution of $X_{t}^{n}=\left(X_{t}^{1, n}, \ldots, X_{t}^{n, n}\right)$, i.e. $\mu_{t}^{n}=\mu_{t}^{n}(\gamma)=\frac{1}{n} \sum_{i=1}^{n} \delta_{X_{t}^{i, n}(\gamma)}$, where $\delta_{y}$ is the Dirac mass at $y$.

Each player $i$ chooses a strategy $\gamma^{i}$, with values in a fixed action space $A$. We assume throughout the whole paper that $A$ is a compact subset of $\mathbb{R}$, hence $A_{\infty}:=\sup _{a \in A}|a|<\infty$. We say that a strategy $\gamma^{i}$ is admissible if it is an $A$-valued predictable process and denote by $\mathbb{G}$ the set of all such strategies. An admissible strategy profile $\gamma$ is an $n$-tuple $\left(\gamma^{1}, \ldots, \gamma^{n}\right) \in \mathbb{G}^{n}$. For player $i$, the expected outcome of the game $G_{n}$ according to the strategy profile $\gamma=\left(\gamma^{1}, \ldots, \gamma^{n}\right)$ is

$$
J^{i, n}(\gamma)=\mathbb{E}\left[\int_{0}^{T} f\left(t, X_{t}^{i, n}(\gamma), \mu_{t}^{n}(\gamma), \gamma_{t}^{i}\right) d t+g\left(X_{T}^{i, n}(\gamma), \mu_{T}^{n}(\gamma)\right)\right]
$$

Each player aims at minimizing the functional $J^{i, n}$ over the set $\mathbb{G}$ of her admissible strategies. We write $X^{i, n}(\gamma)$ and $J^{i, n}(\gamma)$ to stress that both the state variable's dynamics and the expected cost of game $G_{n}$ of player $i$ depend not only on her control $\gamma^{i}$ but also on those of the other players. Given an admissible strategy profile $\gamma=\left(\gamma^{1}, \ldots, \gamma^{n}\right) \in \mathbb{G}^{n}$ and an admissible strategy $\eta \in \mathbb{G},\left(\eta, \gamma_{-i}\right)$ denotes a further admissible strategy where player $i$ deviates from $\gamma$ by playing $\eta$, whereas all the other players keep playing $\gamma^{j}, j \neq i$, i.e. $\left(\eta, \gamma_{-i}\right)=\left(\gamma^{1}, \ldots, \gamma^{i-1}, \eta, \gamma^{i+1}, \ldots, \gamma^{n}\right)$. Our aim is to find an approximate Nash equilibrium for the game $G_{n}$.

Definition 1. Let $\varepsilon \geq 0$. An admissible strategy profile $\gamma=\left(\gamma^{1}, \ldots, \gamma^{n}\right) \in$ $\mathbb{G}^{n}$ is an $\varepsilon$-Nash equilibrium of the $n$-player game $G_{n}$ if for each $i=1, \ldots, n$ 
and for any admissible strategy $\eta \in \mathbb{G}$ the following inequality is satisfied

$$
J^{i, n}\left(\eta, \gamma_{-i}\right) \geq J^{i, n}(\gamma)-\varepsilon
$$

A strategy profile $\gamma$ is a Nash equilibrium in the game $G_{n}$ if it is an $\varepsilon$-Nash equilibrium with $\varepsilon=0$.

\subsection{The associated mean-field game $G_{\infty}$ and main assumptions.}

Let $\left(\Omega, \mathcal{F},\left(\mathcal{F}_{t}\right)_{t \in[0, T]}, P\right)$ be a filtered probability space satisfying the usual conditions and supporting a Brownian motion $W$ and an independent marked point processes $\mu$ with predictable intensity kernel $\nu$ of the form $\nu_{t}(d z)=\lambda_{t} \Phi(d z)$. As before, $\widetilde{\mu}(d z, d t)=\mu(d z, d t)-\nu_{t}(d z) d t$ denotes the compensated jump measure. Let $Y=Y(\gamma)$ be the unique strong solution to

$$
d Y_{t}=b\left(t, Y_{t}, \mu_{t}, \gamma_{t}\right) d t+\sigma\left(t, Y_{t}, \mu_{t}, \gamma_{t}\right) d W_{t}+\int_{\mathbb{R}} \beta\left(t, Y_{t-}, \mu_{t-}, \gamma_{t}, z\right) \widetilde{\mu}(d z, d t)
$$

with initial condition $Y_{0}=\xi \sim \chi$, where $\mu$ is a càdlàg flow of probabilities ${ }^{1}$, $\mu:[0, T] \rightarrow \mathcal{P}(\mathbb{R})$, with $\mu(0-)=\delta_{0}$. The expected outcome of the game when playing some strategy $\gamma$ is defined by

$$
J(\gamma)=\mathbb{E}\left[\int_{0}^{T} f\left(t, Y_{t}(\gamma), \mu_{t}, \gamma_{t}\right) d t+g\left(Y_{T}(\gamma), \mu_{T}\right)\right] .
$$

A MFG solution for $G_{\infty}$ is an admissible process $\widehat{\gamma} \in \mathbb{G}$ which is optimal, i.e. $\widehat{\gamma} \in \arg \min _{\gamma \in \mathbb{G}} J(\gamma)$, and satisfies the mean-field condition $\mu_{t}=\mathcal{L}\left(Y_{t}\right)$ for all $t \in[0, T]$. A solution $\widehat{\gamma}$ of $G_{\infty}$ is said to be Markovian if $\widehat{\gamma}_{t}=\widehat{\gamma}\left(t, Y_{t-}\right)$ where $\widehat{\gamma}$ is a measurable function. For the games $G_{n}$ and $G_{\infty}$ to be well-defined we will be working under the following assumption on

$$
\begin{gathered}
(b, \sigma):[0, T] \times \mathbb{R} \times \mathcal{P}(\mathbb{R}) \times A \rightarrow \mathbb{R}^{2}, \quad \beta:[0, T] \times \mathbb{R} \times \mathcal{P}(\mathbb{R}) \times A \times \mathbb{R} \rightarrow \mathbb{R}, \\
\lambda:[0, T] \rightarrow \mathbb{R}_{+}, \quad f:[0, T] \times \mathbb{R} \times \mathcal{P}(\mathbb{R}) \times A \rightarrow \mathbb{R}, \quad g: \mathbb{R} \times \mathcal{P}(\mathbb{R}) \rightarrow \mathbb{R},
\end{gathered}
$$

where $\mathbb{R}_{+}$denotes the set of all positive real numbers. The following assumption guarantees, in particular, existence of a unique strong solution for the SDEs in eq. (1) and (4) for all admissible strategies.

Assumption 1. 1. $\chi \in \mathcal{P}^{q}(\mathbb{R})$ for some $q>2, q \neq 4$.

\footnotetext{
${ }^{1}$ The limits are taken for the weak convergence of measures.
} 
2. There exists a positive constant $L$ such that for all $t \in[0, T], x, y, z \in$ $\mathbb{R}, \mu, \nu \in \mathcal{P}^{2}(\mathbb{R})$ and $\gamma, \eta \in A$

$$
\begin{gathered}
|(b, \sigma)(t, x, \mu, \gamma)-(b, \sigma)(t, y, \nu, \gamma)|+|\beta(t, x, \mu, \gamma, z)-\beta(t, x, \nu, \eta, z)| \\
\leq L\left(|x-y|+d_{W, 2}(\mu, \nu)+|\gamma-\eta|\right) .
\end{gathered}
$$

Moreover, $b$ and $\sigma$ have at most linear growth (uniformly in $t$ ), $\beta$ has at most linear growth (uniformly in $(t, z)$ ) and $\lambda$ is bounded and Lipschitz-continuous.

3. For all $x, y \in \mathbb{R}, \mu, \nu \in \mathcal{P}^{2}(\mathbb{R}), t \in[0, T]$, and $\gamma \in A$, we have

$$
|f(t, x, \mu, \gamma)-f(t, y, \nu, \gamma)|+|g(x, \mu)-g(y, \nu)| \leq L\left(|x-y|+d_{W, 2}(\mu, \nu)\right) .
$$

Moreover, $f$ and $g$ have at most quadratic growth (uniformly in $t$ ).

Here $d_{W, 2}$ stands for the squared Wasserstein distance, while $\|\cdot\|_{\infty}$ denotes the sup-norm. From now on, to simplify the notation, we write $d_{W}$ for $d_{W, 2}$. We observe that the assumption $q \neq 4$ grants the applicability of [8, Th. 1] to obtain the rate of convergence (see our Remark 3 for details). We conclude this part with some classical estimates for the second moment of the process $X^{n}$ (as in (1)) and the corresponding empirical measure flow $\mu^{n}$. The proof is based on standard applications of Burkholder-Davis-Gundy's and Gronwall's inequalities. It is therefore omitted.

Lemma 2.1. Let Assumption 1 hold. Then, for each admissible strategy $\gamma \in \mathbb{G}^{n}$, the processes $X^{i, n}=X^{i, n}(\gamma), i=1, \ldots, n$, solving (1), satisfy

$$
\mathbb{E}\left[\sup _{t \in[0, T]}\left|X_{t}^{i, n}\right|^{2}\right]+\mathbb{E}\left[\sup _{t \in[0, T]} d_{W}\left(\mu_{t}^{n}, \delta_{0}\right)^{2}\right] \leq \widehat{C}\left(1+\mathbb{E}\left[\xi_{1}^{2}\right]\right),
$$

for some constant $\widehat{C}=\widehat{C}\left(\chi, T, M, A_{\infty}\right)$ independent of $n$ and $\gamma$.

\section{Markovian $\varepsilon-$ Nash equilibrium}

This section presents the main result of this paper on how to construct approximate equilibria for the $n$-player game $G_{n}$ provided that the MFG $G_{\infty}$ admits a Markovian solution, $\widehat{\gamma}_{t}=\widehat{\gamma}\left(t, Y_{t-}\right)$. Consider the game $G_{n}$, where each player $i$ implements the optimal strategy function $(t, x) \mapsto \widehat{\gamma}(t, x)$ evaluated at the left-limit of her own state process $\widehat{X}_{t-}^{i, n}$, i.e. $\widehat{\gamma}_{t}^{i}=\widehat{\gamma}\left(t, \widehat{X}_{t-}^{i, n}\right)$. The $n$-tuple $\widehat{X}^{n}=\left(\widehat{X}^{1, n}, \ldots, \widehat{X}^{n, n}\right)$ is defined as solution to the SDE

$$
\begin{aligned}
d \widehat{X}_{t}^{i, n}= & b\left(t, \widehat{X}_{t}^{i, n}, \mu_{t}^{n}, \widehat{\gamma}\left(t, \widehat{X}_{t-}^{i, n}\right)\right) d t+\sigma\left(t, \widehat{X}_{t}^{i, n}, \mu_{t}^{n}, \widehat{\gamma}\left(t, \widehat{X}_{t-}^{i, n}\right)\right) d W_{t}^{i} \\
& +\int_{\mathbb{R}} \beta\left(t, \widehat{X}_{t-}^{i, n}, \mu_{t-}^{n}, \widehat{\gamma}\left(t, \widehat{X}_{t-}^{i, n}\right), z\right) \widetilde{\mu}^{i}(d z, d t),
\end{aligned}
$$


with $\widehat{X}_{0}^{i, n}=\xi^{i}$. For each player the strategy $\widehat{\gamma}\left(t, \widehat{X}_{t-}^{i, n}\right)$ is admissible, i.e. $\left(\widehat{\gamma}\left(t, \widehat{X}_{t-}^{i, n}\right)\right)_{t \in[0, T]} \in \mathbb{G}$, being $\widehat{\gamma}$ a measurable function by construction and $\widehat{X}_{t-}^{i, n}$ a predictable process. The results of this section are proved under the following standing assumption:

Assumption 2. There exists a Markovian MFG solution $\widehat{\gamma}_{t}=\widehat{\gamma}\left(t, Y_{t-}\right)$ for the game $G_{\infty}$, for some measurable function $\widehat{\gamma}:[0, T] \times \mathbb{R} \rightarrow A$, which is Lipschitz continuous in $x$ (uniformly in $t$ ) with Lipschitz constant $C_{\widehat{\gamma}}$.

Remark 1. The Lipschitz continuity of the MFG solution is typically satisfied, for instance, in the linear-quadratic case when the marked point process is a Poisson process with constant intensity (as in [2]). Indeed in this case the optimal control would be a time-dependent linear function of $x$, suitably truncated to keep it in $A$.

Now, we can state the main result of this paper.

Theorem 3.1. Let Assumptions 1 and 2 hold and let $\widehat{X}^{n}$ be the solution of the SDE (7). Hence the $n$-tuple $\left(\widehat{\gamma}\left(t, \widehat{X}_{t-}^{1, n}\right), \ldots, \widehat{\gamma}\left(t, \widehat{X}_{t-}^{n, n}\right)\right)$ is an $\varepsilon_{n}-$ Nash equilibrium for the $n$-player game $G_{n}$, with $\varepsilon_{n}=O\left(n^{-\alpha / 2}\right) \rightarrow 0$ as $n \rightarrow \infty$, where $\alpha=\min \{1 / 2,(q-2) / q\}$.

Without loss of generality we can assume that $C_{\widehat{\gamma}}=L$ as in Assumption 1. Moreover, from now on the strategy profile $\left(\widehat{\gamma}\left(t, \widehat{X}_{t-}^{1, n}\right), \ldots, \widehat{\gamma}\left(t, \widehat{X}_{t-}^{n, n}\right)\right)$, $t \in[0, T]$, will be shortly denoted by $\widehat{\gamma}^{n}$.

Notice that since the game $G_{n}$ is symmetric, in the proof of Th. 3.1 it will suffice to consider deviations of player 1 only. More precisely, we will focus on two different scenarios: the case when all the players follow the recipe suggested by $G_{\infty}$, i.e. they all play $\widehat{\gamma}\left(t, \widehat{X}_{t-}^{i, n}\right)$, and the case when player 1 deviates to a different strategy $\eta \in \mathbb{G}$, i.e. the strategy profile is $\left(\eta, \widehat{\gamma}_{-1}^{n}\right)=$ $\left(\left(\eta_{t}, \widehat{\gamma}\left(t, \widehat{X}_{t-}^{2, n}\right), \ldots, \widehat{\gamma}\left(t, \widehat{X}_{t-}^{n, n}\right)\right)\right)_{t \in[0, T]}$. In what follows, the strategy $\left(\eta, \widehat{\gamma}_{-1}^{n}\right)$ will be simply denoted by $\eta^{\widehat{\gamma}}$ and the solution of (1) under such a strategy will be denoted by $\widetilde{X}^{i, n}$. We will also need the processes $Y^{i, n}=Y^{i, n}(\widehat{\gamma})$ and $\widetilde{Y}^{1, n}=\widetilde{Y}^{1, n}(\eta)$, which are solutions of the following SDEs:

$$
\begin{aligned}
d Y_{t}^{i, n}= & b\left(t, Y_{t}^{i, n}, \widehat{\mu}_{t}, \widehat{\gamma}\left(t, Y_{t-}^{i, n}\right)\right) d t+\sigma\left(t, Y_{t}^{i, n}, \widehat{\mu}_{t}, \widehat{\gamma}\left(t, Y_{t-}^{i, n}\right)\right) d W_{t}^{i} \\
& +\int_{\mathbb{R}} \beta\left(t, Y_{t-}^{i, n}, \widehat{\mu}_{t-}, \widehat{\gamma}\left(t, Y_{t-}^{i, n}\right), z\right) \widetilde{\mu}^{i}(d z, d t), \\
d \widetilde{Y}_{t}^{1, n}= & b\left(t, \widetilde{Y}_{t}^{1, n}, \widehat{\mu}_{t}, \eta_{t}\right) d t+\sigma\left(t, \widetilde{Y}_{t}^{1, n}, \widehat{\mu}_{t}, \eta_{t}\right) d W_{t}^{1} \\
& +\int_{\mathbb{R}} \beta\left(t, \widetilde{Y}_{t-}^{1, n}, \widehat{\mu}_{t-}, \eta_{t}\right) \widetilde{\mu}^{1}(d z, d t),
\end{aligned}
$$


with initial conditions, respectively, $Y_{0}^{i, n}=\xi^{i}$ and $\widetilde{Y}_{0}^{1, n}=\xi^{1}$, and where $\widehat{\mu}_{t}$ is the law of the state process of the limiting game $G_{\infty}$ under $\widehat{\gamma}$. Since, for each $i$, the process $Y^{i, n}$ has the same dynamics as the representative player's state in $G_{\infty}$ (cf. eq. (4)), and $\widehat{\gamma}$ is a Markovian MFG solution, we have $\mathcal{L}\left(Y_{t}^{i, n}\right)=\widehat{\mu}_{t}$ for all $t \in[0, T]$.

Remark 2. The definition of the processes $Y^{i, n}$ and $\tilde{Y}^{i, n}$ differs from those of $\widehat{X}^{i, n}$ and $\widetilde{X}^{i, n}$ only in the different measure appearing in their SDEs. Indeed in (1), the dynamics of $X^{i, n}$ evolves under the empirical distribution of $X^{n}$, i.e. $\mu^{n}$, while those of $Y^{i, n}$ and $\widetilde{Y}^{1, n}$ in (8) and (9) evolve under $\widehat{\mu}$. This implies that $Y^{i, n}$ and $\widetilde{Y}^{1, n}$ do no longer depend on the other players' choices (and we will say that they do not depend on $n$, for short).

Remark 3. Let $\mu_{t}^{Y, n}=\mu_{t}^{Y, n}(\widehat{\gamma})=\frac{1}{n} \sum_{i=1}^{n} \delta_{Y_{t}^{i, n}}$. As first step we show

$$
\lim _{n \rightarrow \infty} \mathbb{E}\left[d_{W}\left(\widehat{\mu}_{t}, \mu_{t}^{Y, n}\right)^{2}\right]=0
$$

Being $Y_{t}^{i, n}$ independent, identically $\widehat{\mu}_{t}$-distributed random variables, [8, Th.1] implies $\mathbb{E}\left[d_{W}\left(\widehat{\mu}_{t}, \widehat{\mu}_{t}^{Y, n}\right)^{2}\right] \leq C(q) M_{q}^{2 / q}(\widehat{\mu})\left(1 / \sqrt{n}+1 / n^{\frac{q-2}{q}}\right)$, where $C>0$ is a constant depending on $q$, and $M_{q}(\mu)=\int_{\mathbb{R}}|x|^{q} \mu(d x)$. Moreover, arguing as in Lemma 2.1, while relying on the stronger property $\chi \in \mathcal{P}^{q}(\mathbb{R})$ with $q>2$, we have $\mathbb{E}\left[\sup _{t \in[0, T]}\left|Y_{t}\right|^{q}\right] \leq \widehat{C}_{2}(\chi, T, M)$, so that $M_{q}(\widehat{\mu})<\infty$. Hence $\mathbb{E}\left[d_{W}\left(\widehat{\mu}_{t}, \mu_{t}^{Y, n}\right)^{2}\right]=O\left(n^{-\alpha}\right)$, with $\alpha=\min \{1 / 2,(q-2) / q\}$, and, since $q>2$, eq. (10) holds uniformly in time.

Now, we show that the process $Y^{i, n}(\widehat{\gamma})$ approximates $\widehat{X}^{i, n}$ as $n$ is large, in a sense that will be specified later. Note that being independent of $n$, the dynamics of $Y^{i, n}(\widehat{\gamma})$ is easier to study. We recall that in both systems $\widehat{X}^{n}$ and $Y^{n}$, all $n$ players choose a strategy of the same form, i.e. $\widehat{\gamma}\left(t, \widehat{X}_{t-}^{i, n}\right)$ and $\widehat{\gamma}\left(t, Y_{t-}^{i, n}\right)$.

Proposition 3.2. Let $\widehat{X}^{i, n}$ and $Y^{i, n}$ be defined as in eq. (7) and (8), respectively. Then we have

$$
\sup _{t \in[0, T]}\left\|d_{W}\left(\mu_{t}^{n}, \widehat{\mu}_{t}\right)\right\|_{L^{2}}^{2}+\sup _{t \in[0, T]}\left\|\widehat{X}_{t}^{i, n}-Y_{t}^{i, n}\right\|_{L^{2}}^{2}=O\left(n^{-\alpha}\right) .
$$

Proof. Let $b_{t}^{\widehat{X}, i, n}:=b\left(t, \widehat{X}_{t}^{i, n}, \mu_{t}^{n}, \widehat{\gamma}\left(t, \widehat{X}_{t}^{i, n}\right)\right), b_{t}^{Y, i, n}:=b\left(t, Y_{t}^{i, n}, \widehat{\mu}_{t}, \widehat{\gamma}\left(t, Y_{t}^{i, n}\right)\right)$, 
and similarly for the other coefficients. For each $t \in[0, T]$ we have

$$
\begin{aligned}
\left\|\widehat{X}_{t}^{i, n}-Y_{t}^{i, n}\right\|_{L^{2}}^{2} \leq & 3 t \int_{0}^{t}\left\|b_{t}^{\widehat{X}, i, n}-b_{t}^{Y, i, n}\right\|_{L^{2}}^{2} d s+3 \int_{0}^{t}\left\|\sigma_{t}^{\widehat{X}, i, n}-\sigma_{t}^{Y, i, n}\right\|_{L^{2}}^{2} d s \\
& +3 \int_{0}^{t} \int_{\mathbb{R}}\left\|\beta_{t}^{\widehat{X}, i, n}(z)-\beta_{t}^{Y, i, n}(z)\right\|_{L^{2}}^{2} \Phi(d z) \lambda_{s} d s .
\end{aligned}
$$

Using the Lipschitz continuity of $b, \sigma, \beta$ (cf. Assumption 1.2) and of $\widehat{\gamma}(t, \cdot)$, as well as the finiteness of $\mathbb{E}\left[\sup _{t \in[0, T]} d_{W}\left(\mu_{t}^{n}, \delta_{0}\right)^{2}\right]$ in $(6)$, we obtain

$$
\begin{aligned}
\left\|\widehat{X}_{t}^{i, n}-Y_{t}^{i, n}\right\|_{L^{2}}^{2} & \leq 3 L^{2} \int_{0}^{t}\left((2 t+1)\left\|\widehat{X}_{s}^{i, n}-Y_{s}^{i, n}\right\|_{L^{2}}^{2}+2 t\left\|d_{W}\left(\mu_{s}^{n}, \widehat{\mu}_{s}\right)\right\|_{L^{2}}^{2}\right) d s \\
+6 L^{2} & \|\lambda\|_{\infty} \int_{0}^{t}\left(\left\|d_{W}\left(\mu_{s}^{n}, \widehat{\mu}_{s}\right)\right\|_{L^{2}}^{2}+\left\|\widehat{\gamma}\left(s, \widehat{X}_{s}^{i, n}\right)-\widehat{\gamma}\left(s, Y_{s}^{i, n}\right)\right\|_{L^{2}}^{2}\right) d s \\
& \leq \widetilde{C} \int_{0}^{t}\left(\left\|\widehat{X}_{s}^{i, n}-Y_{s}^{i, n}\right\|_{L^{2}}^{2}+\left\|d_{W}\left(\mu_{s}^{n}, \widehat{\mu}_{s}\right)\right\|_{L^{2}}^{2}\right) d s,
\end{aligned}
$$

for a suitable constant $\widetilde{C}=\widetilde{C}(T, L, M)$. Moreover, inequality (12) yields

$$
\begin{aligned}
\left\|d_{W}\left(\mu_{t}^{n}, \mu_{t}^{Y, n}\right)\right\|_{L^{2}}^{2} & \leq \frac{1}{n} \sum_{i=1}^{n}\left\|\widehat{X}_{t}^{i, n}-Y_{t}^{i, n}\right\|_{L^{2}}^{2} \\
& \leq \frac{\widetilde{C}}{n} \sum_{i=1}^{n} \int_{0}^{t}\left(\left\|\widehat{X}_{s}^{i, n}-Y_{s}^{i, n}\right\|_{L^{2}}^{2}+\left\|d_{W}\left(\mu_{s}^{n}, \widehat{\mu}_{s}\right)\right\|_{L^{2}}^{2}\right) d s .
\end{aligned}
$$

Then, it holds that

$$
\begin{aligned}
& \left\|d_{W}\left(\mu_{t}^{n}, \widehat{\mu}_{t}\right)\right\|_{L^{2}}^{2}+\frac{1}{n} \sum_{i=1}^{n}\left\|\widehat{X}_{t}^{i, n}-Y_{t}^{i, n}\right\|_{L^{2}}^{2} \\
& \quad \leq 2\left\|d_{W}\left(\mu_{t}^{n}, \mu_{t}^{Y, n}\right)\right\|_{L^{2}}^{2}+2\left\|d_{W}\left(\widehat{\mu}_{t}, \mu_{t}^{Y, n}\right)\right\|_{L^{2}}^{2}+\frac{1}{n} \sum_{i=1}^{n}\left\|\widehat{X}_{t}^{i, n}-Y_{t}^{i, n}\right\|_{L^{2}}^{2} \\
& \quad \leq 2\left\|d_{W}\left(\widehat{\mu}_{t}, \mu_{t}^{Y, n}\right)\right\|_{L^{2}}^{2}+(2 \widetilde{C}+1) \int_{0}^{t}\left(\left\|d_{W}\left(\mu_{s}^{n}, \widehat{\mu}_{s}\right)\right\|_{L^{2}}^{2}+\frac{1}{n} \sum_{i=1}^{n}\left\|\widehat{X}_{s}^{i, n}-Y_{s}^{i, n}\right\|_{L^{2}}^{2}\right) d s .
\end{aligned}
$$

Therefore, by eq. (10) and Remark 3, we have

$$
\begin{aligned}
& \left\|d_{W}\left(\mu_{t}^{n}, \widehat{\mu}_{t}\right)\right\|_{L^{2}}^{2}+\frac{1}{n} \sum_{i=1}^{n}\left\|\widehat{X}_{t}^{i, n}-Y_{t}^{i, n}\right\|_{L^{2}}^{2} \\
& \quad \leq O\left(n^{-\alpha}\right)+2 \widetilde{C} \int_{0}^{t}\left(\left\|d_{W}\left(\mu_{s}^{n}, \widehat{\mu}_{s}\right)\right\|_{L^{2}}^{2}+\frac{1}{n} \sum_{i=1}^{n}\left\|\widehat{X}_{s}^{i, n}-Y_{s}^{i, n}\right\|_{L^{2}}^{2}\right) d s,
\end{aligned}
$$


so that Gronwall's lemma applies, giving eq. (11).

In the previous estimates, we have considered the case when all $n$ players are choosing the same strategy $\widehat{\gamma}$. We now study what happens when player 1 deviates from the strategy profile $\widehat{\gamma}^{n}$ by playing $\eta \in \mathbb{G}$. In this case the dynamics of each player in $G_{n}$ is given by the solution to (1), under the strategy $\eta^{\widehat{\gamma}}$, i.e. $\widetilde{X}^{i, n}$.

Proposition 3.3. Let $\widehat{X}$ and $\widetilde{X}$ be the solutions of the system (1), when the strategy profile is given by $\widehat{\gamma}^{n}$ and $\eta^{\widehat{\gamma}}$, respectively. We denote by $\mu^{n}$ and $\widetilde{\mu}^{n}$ the empirical distribution of the two systems. Then,

$$
\sup _{t \in[0, T]}\left\|d_{W}\left(\mu_{t}^{n}, \widetilde{\mu}_{t}^{n}\right)\right\|_{L^{2}}^{2}=O\left(n^{-1}\right) .
$$

Moreover, for the process $\widetilde{Y}^{1, n}$ defined in eq. (9), we have

$$
\sup _{t \in[0, T], \eta \in \mathbb{G}}\left\|\widetilde{X}_{t}^{1, n}-\widetilde{Y}_{t}^{1, n}\right\|_{L^{2}}^{2}=O\left(n^{-\alpha}\right) .
$$

Proof. First, let us consider player 1. By Lemma 2.1, the Lipschitz continuity of $b, \sigma$ and $\beta$, and the boundedness of $\lambda$ (cf. Assumption 1.2) imply

$$
\begin{aligned}
\left\|\widehat{X}_{t}^{1, n}-\widetilde{X}_{t}^{1, n}\right\|_{L^{2}}^{2} \leq & 3 L^{2} \int_{0}^{t}\left((2 t+1)\left\|\widehat{X}_{s}^{1, n}-\widetilde{X}_{s}^{1, n}\right\|_{L^{2}}^{2}+2 t\left\|d_{W}\left(\mu_{s}^{n}, \widetilde{\mu}_{s}^{n}\right)\right\|_{L^{2}}^{2}\right) d s \\
& +3 \mathbb{E}\left[\int_{0}^{t}\left(L d_{W}\left(\mu_{s}^{n}, \widetilde{\mu}_{s}^{n}\right)+L\left|\widehat{\gamma}\left(s, \widehat{X}_{s}^{1, n}\right)-\eta_{s}\right|\right)^{2} \lambda(s) d s\right] \\
\leq & 12 L^{2} T\left(\left(4 T+2\|\lambda\|_{\infty}+1\right) \widehat{C}\left(1+\mathbb{E}\left[\xi_{1}^{2}\right]\right)+A_{\infty}^{2}\|\lambda\|_{\infty}\right)=: C_{1},
\end{aligned}
$$

where recall that the constant $\widehat{C}$, given as in eq. (6), is independent of $n$ and then so is $C_{1}$. Furthermore, by definition $C_{1}$ does not depend on $\eta$ either. On the other hand, the other players $i=2, \ldots, n$ play the strategy $\widehat{\gamma}\left(t, X_{t-}^{i, n}\right)$ in both cases, then, to find an estimate for $\left\|\widehat{X}_{t}^{i, n}-\widetilde{X}_{t}^{i, n}\right\|_{L^{2}}^{2}$ we can argue as for (12). Following the same ideas leading to (13), we have that

$$
\begin{aligned}
\left\|d_{W}\left(\mu_{t}^{n}, \widetilde{\mu}_{t}^{n}\right)\right\|_{L^{2}}^{2} & \leq \frac{1}{n}\left\|\widehat{X}_{t}^{1, n}-\widetilde{X}_{t}^{1, n}\right\|_{L^{2}}^{2}+\frac{1}{n} \sum_{i=2}^{n}\left\|\widehat{X}_{t}^{i, n}-\widetilde{X}_{t}^{i, n}\right\|_{L^{2}}^{2} \\
& \leq \frac{C_{1}}{n}+\frac{\widetilde{C}}{n} \sum_{i=2}^{n} \int_{0}^{t}\left(\left\|\widehat{X}_{s}^{i, n}-\widetilde{X}_{s}^{i, n}\right\|_{L^{2}}^{2}+\left\|d_{W}\left(\mu_{s}^{n}, \widetilde{\mu}_{s}^{n}\right)\right\|_{L^{2}}^{2}\right) d s
\end{aligned}
$$


and therefore

$$
\begin{aligned}
& \left\|d_{W}\left(\mu_{t}^{n}, \widetilde{\mu}_{t}^{n}\right)\right\|_{L^{2}}^{2}+\frac{1}{n} \sum_{i=2}^{n}\left\|\widehat{X}_{t}^{i, n}-\widetilde{X}_{t}^{i, n}\right\|_{L^{2}}^{2} \\
& \leq \frac{C_{1}}{n}+\frac{2 \widetilde{C}}{n} \sum_{i=2}^{n} \int_{0}^{t}\left(\left\|\widehat{X}_{s}^{1, n}-\widetilde{X}_{s}^{1, n}\right\|_{L^{2}}^{2}+\left\|d_{W}\left(\mu_{s}^{n}, \widetilde{\mu}_{s}^{n}\right)\right\|_{L^{2}}^{2}\right) d s .
\end{aligned}
$$

Applying Gronwall's lemma, we have

$$
\left\|d_{W}\left(\mu_{t}^{n}, \widetilde{\mu}_{t}^{n}\right)\right\|_{L^{2}}^{2}+\frac{1}{n} \sum_{i=2}^{n}\left\|\widehat{X}_{t}^{i, n}-\widetilde{X}_{t}^{i, n}\right\|_{L^{2}}^{2} \leq \frac{K_{1}}{n},
$$

with $K_{1}=K_{1}\left(\chi, T, L, M, A_{\infty}\right)$ a constant independent of $n, t$ and $\eta$, so that (14) is proved. Lastly, as in the proof of Prop. 3.2, by considering $\widetilde{Y}^{1, n}$ as defined in (9) we have

$$
\begin{aligned}
\left\|\widetilde{X}_{t}^{1, n}-\widetilde{Y}_{t}^{1, n}\right\|_{L^{2}}^{2} \leq & 3(2 t+1) L^{2} \int_{0}^{t}\left\|\widehat{X}_{s}^{1, n}-Y_{s}^{1, n}\right\|_{L^{2}}^{2} d s \\
& +3 L^{2}\left(2 t+\|\lambda\|_{\infty}\right) \int_{0}^{t}\left\|d_{W}\left(\mu_{s}^{n}, \widehat{\mu}_{s}\right)\right\|_{L^{2}}^{2} d s \\
\leq & \widetilde{K} \int_{0}^{t}\left(\left\|\widetilde{X}_{s}^{1, n}-\widetilde{Y}_{s}^{1, n}\right\|_{L^{2}}^{2}+\left\|d_{W}\left(\mu_{s}^{n}, \widehat{\mu}_{s}\right)\right\|_{L^{2}}^{2}\right) d s
\end{aligned}
$$

therefore for a suitable constant $\widetilde{K}=\widetilde{K}(T, L, M)$ we have

$$
\left\|\widetilde{X}_{t}^{1, n}-\widetilde{Y}_{t}^{1, n}\right\|_{L^{2}}^{2} \leq \widetilde{K} \int_{0}^{t}\left\|\widetilde{X}_{s}^{1, n}-\widetilde{Y}_{s}^{1, n}\right\|_{L^{2}}^{2} d s+\widetilde{K} O\left(n^{-\alpha}\right)
$$

Hence one more application of Gronwall's lemma gives

$$
\left\|\widetilde{X}_{t}^{1, n}-\widetilde{Y}_{t}^{1, n}\right\|_{L^{2}}^{2} \leq \bar{K} O\left(n^{-\alpha}\right),
$$

for some further constant $\bar{K}=\bar{K}(T, L, M)$ independent of $n, t$ and $\eta$, whence eq. (15).

Remark 4. It is crucial here and in the following that the constants $K_{1}$ and $\bar{K}$ appearing in (16) and (17) do not depend on how player 1 deviates from the strategy profile $\widehat{\gamma}^{n}$. 
To complete the proof of Th. 3.1, we introduce the operators $\widetilde{J}_{n}: \mathbb{G}^{n} \rightarrow \mathbb{R}$ and $\widetilde{J}: \mathbb{G} \rightarrow \mathbb{R}$ as

$$
\begin{aligned}
\widetilde{J}_{n}(\gamma) & =\mathbb{E}\left[\int_{0}^{T} f\left(t, X_{t}^{1, n}(\gamma), \widehat{\mu}_{t}, \gamma_{t}^{1}\right) d t+g\left(X_{T}^{1, n}(\gamma), \widehat{\mu}_{T}\right)\right], \\
\widetilde{J}(\eta) & =\mathbb{E}\left[\int_{0}^{T} f\left(t, \widetilde{Y}_{t}^{1, n}, \widehat{\mu}_{t}, \eta_{t}\right) d t+g\left(\widetilde{Y}_{T}^{1, n}(\eta), \widehat{\mu}_{T}\right)\right]
\end{aligned}
$$

respectively, with $X^{1, n}$ and $\widetilde{Y}^{1, n}$ as in (1) and (9). Observe that $\widetilde{J}$ does not depend on the number of players, $n$, in the game. Indeed, $\widetilde{Y}^{1, n}$ follows the dynamics of a representative player in the MFG $G_{\infty}$, and therefore, $\widetilde{J}$ is exactly the expected cost of the strategy $\eta$ in $G_{\infty}$ w.r.t. the flow of measures $\widehat{\mu}$, as in (5). Therefore, since $\widehat{\gamma}\left(t, \widetilde{Y}_{t}^{1, n}\right)$ is by construction a minimizer,

$$
\widetilde{J}\left(\widehat{\gamma}\left(\cdot, \tilde{Y}_{\cdot-}^{1, n}\right)\right) \leq \widetilde{J}(\eta) \text { for all } \eta \in \mathbb{G} .
$$

Now, we show that the value of player 1 in the game $G_{n}$, when he deviates from the candidate Nash equilibrium $\widehat{\gamma}^{n}$ to a different strategy $\eta \in \mathbb{G}$, i.e. $J^{1, n}\left(\eta^{\widehat{\gamma}}\right)$ as in (2), can be approximated, for $n$ large, by $\widetilde{J}_{n}\left(\eta^{\widehat{\gamma}}\right)$.

Proposition 3.4. Let $(t, x) \mapsto \widehat{\gamma}(t, x)$ be as in Assumption 2. Consider the strategy profile $\widehat{\gamma}_{t}^{n}=\left(\widehat{\gamma}\left(t, \widehat{X}_{t-}^{1, n}\right), \ldots, \widehat{\gamma}\left(t, \widehat{X}_{t-}^{n, n}\right)\right)$, for $t \in[0, T]$, and let $\eta$ be an admissible strategy in $\mathbb{G}$. Then

$$
\sup _{\eta \in \mathbb{G}}\left|J^{1, n}\left(\eta^{\widehat{\gamma}}\right)-\widetilde{J}_{n}\left(\eta^{\widehat{\gamma}}\right)\right|=O\left(n^{-\alpha / 2}\right) .
$$

Proof. By definitions (18) and (19) and by Assumption 1.3,

$$
\begin{aligned}
& \left|J^{1, n}\left(\eta^{\widehat{\gamma}}\right)-\widetilde{J}_{n}\left(\eta^{\gamma}\right)\right| \leq \mathbb{E}\left[\int_{0}^{T}\left|f\left(t, \widetilde{X}_{t}^{1, n}, \widetilde{\mu}_{t}^{n}, \eta_{t}\right)-f\left(t, \widetilde{X}_{t}^{1, n}, \widehat{\mu}_{t}, \eta_{t}\right)\right| d t\right] \\
& \quad+\mathbb{E}\left[\left|g\left(\widetilde{X}_{T}^{1, n}, \widetilde{\mu}_{T}^{n}\right)-g\left(\widetilde{X}_{T}^{1, n}, \widehat{\mu}_{T}\right)\right|\right] \leq L \int_{0}^{T} \mathbb{E}\left[d_{W}\left(\widetilde{\mu}_{t}^{n}, \widehat{\mu}_{t}\right)\right] d t+L \mathbb{E}\left[d_{W}\left(\widetilde{\mu}_{T}^{n}, \widehat{\mu}_{T}\right)\right] .
\end{aligned}
$$

Hence eq. (21) follows from previous results in Prop. 3.2 and Prop. 3.3, since $\mathbb{E}\left[d_{W}\left(\widetilde{\mu}_{t}^{n}, \widehat{\mu}_{t}\right)\right] \leq\left\|d_{W}\left(\widetilde{\mu}_{t}^{n}, \widehat{\mu}_{t}\right)\right\|_{L^{2}}=O\left(n^{-\frac{\alpha}{2}}\right)$.

As last preliminary result before concluding, we approximate $\widetilde{J}_{n}\left(\eta^{\widehat{\gamma}}\right)$ with $\widetilde{J}(\eta)$, i.e. the expected cost for playing $\eta$ in the MFG $G_{\infty}$.

Proposition 3.5. Let $(t, x) \mapsto \widehat{\gamma}(t, x)$ be as in Assumption 2, let $\widehat{\gamma}_{t}^{n}=$ $\left(\widehat{\gamma}\left(t, \widehat{X}_{t-}^{1, n}\right), \ldots, \widehat{\gamma}\left(t, \widehat{X}_{t-}^{n, n}\right)\right)$, for $t \in[0, T]$, and let $\eta \in \mathbb{G}$ be an admissible strategy. Then

$$
\sup _{\eta \in \mathbb{G}}\left|\widetilde{J}_{n}\left(\eta^{\widehat{\gamma}}\right)-\widetilde{J}(\eta)\right|=O\left(n^{-\alpha / 2}\right) .
$$


Proof. Arguing as in the proof of Prop. 3.4, we have that

$$
\begin{aligned}
& \left|\widetilde{J}_{n}\left(\eta^{\widehat{\gamma}}\right)-\widetilde{J}(\eta)\right| \leq \mathbb{E}\left[\int_{0}^{T}\left|f\left(t, \widetilde{X}_{t}^{1, n}, \widehat{\mu}_{t}, \eta_{t}\right)-f\left(t, \widetilde{Y}_{t}^{1, n}, \widehat{\mu}_{t}, \eta_{t}\right)\right| d t\right] \\
& \quad+\mathbb{E}\left[\left|g\left(\widetilde{X}_{T}^{1, n}, \widehat{\mu}_{T}\right)-g\left(\widetilde{Y}_{T}^{1, n}, \widehat{\mu}_{T}\right)\right|\right] \leq L \int_{0}^{T} \mathbb{E}\left[\left|\widetilde{X}_{t}^{1, n}-\widetilde{Y}_{t}^{1, n}\right|\right] d t+L \mathbb{E}\left[\left|\widetilde{X}_{T}^{1, n}-\widetilde{Y}_{T}^{1, n}\right|\right] .
\end{aligned}
$$

Since by Prop. 3.3 we have $\mathbb{E}\left[\left|\widetilde{X}_{t}^{1, n}\left(\eta^{\widehat{\gamma}}\right)-\widetilde{Y}_{t}^{1, n}(\eta)\right|\right]=O\left(n^{-\alpha / 2}\right)$, then eq. (22) follows.

Proof of Theorem 3.1. Given an admissible strategy $\eta \in \mathbb{G}$, let

$$
\varepsilon_{n}^{1}=4 \sup _{\eta \in \mathbb{G}}\left|J^{1, n}\left(\eta^{\widehat{\gamma}}\right)-\widetilde{J}_{n}\left(\eta^{\widehat{\gamma}}\right)\right|, \quad \varepsilon_{n}^{2}=4 \sup _{\eta \in \mathbb{G}}\left|\widetilde{J}_{n}\left(\eta^{\widehat{\gamma}}\right)-\widetilde{J}(\eta)\right|, \quad \varepsilon_{n}=\varepsilon_{n}^{1}+\varepsilon_{n}^{2} .
$$

Then $J^{1, n}\left(\eta^{\widehat{\gamma}}\right) \geq-\frac{\varepsilon_{n}}{2}+\widetilde{J}(\eta) \geq-\frac{\varepsilon_{n}}{2}+\widetilde{J}(\widehat{\gamma}) \geq-\varepsilon_{n}+J^{1, n}(\widehat{\gamma})$, which gives (3) for player 1 . The first and the third inequalities are both guaranteed by Prop. 3.4 and 3.5, whereas the second one is justified by eq. (20). The symmetry of the game $G_{n}$ guarantees that $\left(\widehat{\gamma}\left(t, X_{t-}^{1, n}\right), \ldots, \widehat{\gamma}\left(t, X_{t-}^{n, n}\right)\right)$, for $t \in[0, T]$, is an $\varepsilon_{n}$-Nash equilibrium. The rate of convergence, i.e. $\varepsilon_{n}=$ $O\left(n^{-\alpha / 2}\right)$, is also granted by the previous approximations in Prop. 3.4 and 3.5 .

\section{Acknowledgements}

L. Campi and L. Di Persio wish to thank the FBK - CIRM for funding their "Research in Pairs" project "McKean-Vlasov dynamics with Lévy noise with applications to systemic risk" (2015) with the active participation of C. Benazzoli. L. Di Persio and C. Benazzoli also thank the GNAMPA for funding the projects "Stochastic Partial Differential Equations and Stochastic Optimal Transport with Applications to Mathematical Finance" (coord. L. Di Persio, 2016), and "Metodi di controllo ottimo stocastico per l'analisi di problem debt management" (coord. A. Marigonda, 2017).

\section{Bibliography}

[1] Andreis, L., Dai Pra, P., Fischer, M., 2018. McKean-Vlasov limit for interacting systems with simultaneous jumps. Stochastic Analysis and Applications, forthcoming.

[2] Benazzoli, C., Campi, L., Di Persio, L., 2017. Mean-field games with controlled jumps. arXiv preprint arXiv:1703.01919. 
[3] Carmona, R., Delarue, F., 2013. Mean field forward-backward stochastic differential equations. Electron. Commun. Probab 18 (68), 15.

[4] Carmona, R., Delarue, F., 2013. Probabilistic analysis of mean-field games. SIAM Journal on Control and Optimization 51 (4), 2705-2734.

[5] Carmona, R., Delarue, F., 2018. Probabilistic Theory of Mean Field Games with Applications I-II. Springer.

[6] Carmona, R., Lacker, D., 2015. A probabilistic weak formulation of mean field games and applications. The Annals of Applied Probability 25 (3), 1189-1231.

[7] Cecchin, A., Fischer, M., 2018. Probabilistic approach to finite state mean field games. Applied Mathematics \& Optimization, forthcoming.

[8] Fournier, N., Guillin, A., 2015. On the rate of convergence in Wasserstein distance of the empirical measure. Probability Theory and Related Fields 162 (34), $707-738$.

[9] Gomes, D. A., Mohr, J., Souza, R. R., 2013. Continuous time finite state mean field games. Applied Mathematics \& Optimization 68 (1), 99-143.

[10] Graham, C., 1992. McKean-Vlasov Itô-Skorohod equations, and nonlinear diffusions with discrete jump sets. Stochastic processes and their applications $40(1), 69-82$.

[11] Guéant, O., Lasry, J.-M., Lions, P.-L., 2011. Mean field games and applications. In: Paris-Princeton lectures on mathematical finance 2010. Springer, pp. $205-266$.

[12] Hafayed, M., Abba, A., Abbas, S., 2014. On mean-field stochastic maximum principle for near-optimal controls for Poisson jump diffusion with applications. International Journal of Dynamics and Control 2 (3), 262-284.

[13] Huang, M., Malhamé, R. P., Caines, P. E., 2006. Large population stochastic dynamic games: closed-loop McKean-Vlasov systems and the Nash certainty equivalence principle. Communications in Information \& Systems 6 (3), 221252 .

[14] Jourdain, B., Méléard, S., Woyczynski, W., 2008. Nonlinear SDEs driven by Lévy processes and related PDEs. Alea 4, 1-29.

[15] Kolokoltsov, V. N., Li, J., Yang, W., 2011. Mean field games and nonlinear Markov processes. arXiv preprint arXiv:1112.3744.

[16] Lasry, J.-M., Lions, P.-L., 2006. Jeux à champ moyen. I-Le cas stationnaire. Comptes Rendus Mathématique 343 (9), 619-625. 
[17] Lasry, J.-M., Lions, P.-L., 2006. Jeux à champ moyen. II-Horizon fini et contrôle optimal. Comptes Rendus Mathématique 343 (10), 679-684.

[18] Lasry, J.-M., Lions, P.-L., 2007. Mean field games. Japanese Journal of Mathematics 2 (1), 229-260. 